\title{
Lipid desaturation-associated endoplasmic reticulum stress regulates MYCN gene expression in hepatocellular carcinoma cells
}

\author{
Xian-Yang Qin ${ }^{1}$, Ting Su ${ }^{1,2}$, Wenkui Yu ${ }^{2}$ and Soichi Kojima ${ }^{1}$
}

\begin{abstract}
Hepatocellular carcinoma (HCC) is the second leading cause of cancer-related deaths worldwide due to its high rate of recurrence, in part because of cancer stem cell (CSC)-dependent "field cancerization". Recently, we identified that the oncogene v-myc avian myelocytomatosis viral oncogene neuroblastoma derived homolog (MYCN) marked CSC-like subpopulations in heterogeneous HCC and served as a therapeutic target and prognostic marker for HCC. In this study, we explored the molecular basis of upregulated MYCN gene expression in HCC cells. Liquid chromatograph time-of-flight mass spectrometry-based metabolome analysis demonstrated that the content of unsaturated fatty acids was increased in MYCN high expression (MYCN ${ }^{\text {high }}$ ) CSC-like HCC cells. Inhibition of lipid desaturation using either the chemical inhibitor or siRNA/shRNA against stearoyl-CoA desaturase-1 (SCD1) suppressed cell proliferation as well as MYCN gene expression in MYCN ${ }^{\text {high }}$ HCC cells, grown as both monolayer and spheres. Further mechanistic study using RNA-seq based transcriptome analysis revealed that endoplasmic reticulum (ER) stress related signaling networks such as endocannabinoid cancer inhibition pathway were under the control of SCD1 in MYCN ${ }^{\text {high }}$ HCC cells. Furthermore, the expression of ER stress-inducible transcription suppressor cyclic AMP-dependent transcription factor (ATF3) was downregulated in MYCN ${ }^{\text {high }}$ CSC-like HCC cells and CSC-rich spheroids, which was upregulated by inhibition of lipid desaturation or treatment with acyclic retinoid (ACR). Lipid profiling using NMR spectroscopy revealed that the ACR dramatically reduced the content of unsaturated fatty acids in HCC cells. The chemical inducer of ER stress inhibited MYCN gene expression, while the chemical inhibitor of ER stress or knockdown of ATF3 gene expression partially rescued the suppression of MYCN gene expression by ACR in MYCN ${ }^{\text {high }}$ HCC cells. These data suggested that lipid desaturation-mediated ER stress signaling regulates MYCN gene expression in HCC cells and serves as a promising therapeutic target for the treatment and prevention of HCC.
\end{abstract}

\section{Introduction}

Hepatocellular carcinoma (HCC) is a well-characterized inflammation-driven cancer $^{1}$ and is the second most lethal cancer worldwide due to its poor prognosis ${ }^{2}$. Persistent infections of hepatitis B virus (HBV) and hepatitis $\mathrm{C}$ virus $(\mathrm{HCV})$ are the major risk factors for $\mathrm{HCC}^{3}$.

\footnotetext{
Correspondence: Xian-Yang Qin (xyqin@riken.jp)

'Liver Cancer Prevention Research Unit, RIKEN Cluster for Pioneering Research, Wako, Saitama 351-0198, Japan

2Department of Intensive Care Unit, the Affiliated Drum Tower Hospital,

Medical School of Nanjing University, Nanjing, Jiangsu 210008, China

Deceased: Soichi Kojima

Edited by P. Pinton
}

Advances in antiviral therapy have reduced the risk of developing both $\mathrm{HBV}_{-}^{4}$ and $\mathrm{HCV}$-related $\mathrm{HCC}^{5}$. However, obesity-associated inflammation is responsible for increased death rates for all the cancers ${ }^{6}$. Non-alcoholic steatohepatitis (NASH), which is characterized by continuous hepatocyte death and compensatory prolifera$\operatorname{tion}^{7}$, has attracted much attention and is believed that it will soon be the leading etiology of $\mathrm{HCC}^{8}$.

Lipogenesis is known to be high in individuals with $\mathrm{NASH}$ and its upregulation is associated with high risk and poor prognosis of NASH-related $\mathrm{HCC}^{9}$. Lipid metabolic reprogramming allows the cells to adapt to the

\section{(c) The Author(s) 2020}

(c) (i) Open Access This article is licensed under a Creative Commons Attribution 4.0 International License, which permits use, sharing, adaptation, distribution and reproduction cc) in any medium or format, as long as you give appropriate credit to the original author(s) and the source, provide a link to the Creative Commons license, and indicate if changes were made. The images or other third party material in this article are included in the article's Creative Commons license, unless indicated otherwise in a credit line to the material. If material is not included in the article's Creative Commons license and your intended use is not permitted by statutory regulation or exceeds the permitted use, you will need to obtain permission directly from the copyright holder. To view a copy of this license, visit http://creativecommons.org/licenses/by/4.0/. 
tumor microenvironments. For example, lipogenesis may promote the cell growth and proliferation of MYC-driven cancers ${ }^{10}$, probably by maintaining the rebalanced nutrient supply and demand for cellular hyperproliferation ${ }^{11}$. In addition, lipid accumulation in hepatocytes selectively induces lipotoxicity in intrahepatic $\mathrm{CD}^{+} \mathrm{T}$ cells and promotes HCC development by evading immune surveillance $^{12}$. Furthermore, there is growing evidence about the role of unsaturated fatty acids in tumor initiation by regulating the generation and maintenance of cancer stem cells (CSCs) or tumor-initiating cells (TICs) ${ }^{13}$.

Tissue regeneration and tumorigenesis share common molecular pathways in the regulation of cell growth and death ${ }^{14}$. In response to resection or injury, healthy liver has the unique ability to grow back or regenerate by a process of compensatory hyperplasia ${ }^{15}$. In contrast, under chronic inflammation such as lipid-rich environment, repeated liver injury and compensatory proliferation might lead to aberrant stabilization and chronic activation of the oncogenes, which can be considered as one of the leading causes of HCC. The MYC family members are critically involved in the regulation of multiple biological processes, including cell growth, proliferation, apoptosis, energy metabolism, and differentiation ${ }^{16}$, and play dual roles in regulating both hepatocellular proliferation and hepatocarcinogenesis ${ }^{17}$. $v$-myc avian myelocytomatosis viral oncogene neuroblastoma derived homolog $(M Y C N)$, is a well-recognized oncogene associated with the progression and prognosis of neuroblastoma ${ }^{18}$. A transcriptome analysis performed by our group in primary hepatocytes during the process of mouse liver regeneration revealed that the expression of Mycn was low in normal hepatocytes, but increased along with hepatocyte proliferation after partial hepatectomy ${ }^{19}$. We also reported that MYCN expression was seen in epithelial cell adhesion molecule (EpCAM) ${ }^{+}$liver CSC-like cells and was positively correlated with the recurrence of $\mathrm{HCC}^{20}$. However, the mechanism underlying the overexpression of MYCN during chronic liver injury and hepatic tumorigenesis is still unclear.

Acyclic retinoid (ACR) is a synthetic vitamin A-like compound capable of preventing the recurrence of HCC in patients after curative removal of the primary tumors ${ }^{21}$. Recently, we identified that the MYCN high expression $\left(\mathrm{MYCN}^{\text {high }}\right)$ liver CSC-like cells are selectively depleted by $\mathrm{ACR}$, suggesting $\mathrm{MYCN}$ as a therapeutic target for the prevention and treatment of $\mathrm{HCC}^{20}$. Further proteome analysis showed enrichment in $\mathrm{MYCN}^{\text {high }} \mathrm{EpCAM}^{+} \mathrm{CSC}-$ like HCC cells for lipogenic enzymes such as stearoylCoenzyme A desaturase-1 (SCD1), an enzyme that creates double bonds at specific locations in long chain fatty acids $^{22}$. Therefore, in this study, we examined the link between lipid desaturation and $M Y C N$ gene expression in HCC cells.

\section{Results}

\section{Metabolome characterization of $\mathrm{MYCN}^{\text {high }}$ CSC-like HCC cells}

To determine the metabolic characteristics of $\mathrm{MYCN}^{\text {high }}$ CSC-like HCC cells, metabolite analysis was performed on the $\mathrm{EpCAM}^{+/-} \mathrm{JHH} 7$ cells sorted using fluorescence activated cell sorting (FACS). Peaks of a total of 65 lipophilic metabolites were detected using liquid chromatograph time-of-flight mass spectrometry (LC-TOFMS) (Table S1). Hierarchical cluster analysis (HCA) demonstrated a clear distinction in the abundance of lipophilic metabolites between EpCAM $^{+/-}$cells (Fig. 1a). The pathway impact analysis of the differentially expressed metabolites with a threshold of fold change of more than 2 using MetaboAnalyst showed that linoleic acid (LA; C18:2) metabolism was the most significantly perturbed pathway between $\mathrm{EpCAM}^{+/-}$cells (Fig. 1b). Furthermore, in accordance with the proteome analysis ${ }^{22}$, the content of unsaturated fatty acids was strikingly increased in the $\mathrm{EpCAM}^{+}$cells compared with that in the $\mathrm{EpCAM}^{-}$cells. Palmitoleic acid (PA, C16:1; 6.8-fold) and oleic acid (OA, C18:1; 5.6-fold), which are the main monounsaturated fatty acids generated via $\mathrm{SCD} 1^{23}$, were the two most dramatically enhanced lipophilic metabolites in $\mathrm{EpCAM}^{+}$ cells. In contrast, there were modest increases in the content of saturated fatty acids such as stearic acid (SA, C18:0; 1.6-fold) and palmitic acid (C16:0; 2.1-fold), and almost no changes in cholesterol ( 0.79 -fold) and cholesterol sulfate (1.1-fold) in the EpCAM ${ }^{+}$cells compared with those in the $\mathrm{EpCAM}^{-}$cells (Fig. 1c).

\section{MYCN co-expression genes in HCC tumor tissues and cell lines}

Next, we undertook a clinical investigation of MYCN co-expression genes in human HCC tumor tissues. The genes were selected using The Cancer Genome Atlas (TCGA) database, which contains a RNA-seq dataset of 372 HCC tumor samples (PanCancer Atlas) ${ }^{24}$. With a threshold of Spearman's correlation coefficient of more than 0.3, 109 genes were selected as MYCN co-expression genes and then subjected to pathway analysis (Fig. 2a). Ingenuity Pathway Analysis (IPA) revealed that the top canonical pathways such as the fatty acid activation and $\gamma$ linolenate biosynthesis were correlated with MYCN in HCC (Fig. 2b). Then, we examined the correlation between the MYCN gene expression and well-established hepatic stem/progenitor markers ${ }^{25}$ and genes associated with cancer metabolism and metastasis ${ }^{26}$ by data mining in the Cancer Cell Line Encyclopedia (CCLE) database ${ }^{27}$. With a threshold expression level of MYCN at 1 read per kilobase of exon per million mapped reads (RPKM), 8 out of a total of $25 \mathrm{HCC}$ cell lines were selected as MYCN $\mathrm{N}^{\text {high }}$ cells ( $\geq 1$ RPKM), while the other 17 HCC cell lines were MYCN low expression $\left(\mathrm{MYCN}^{\text {low }}\right)$ cells $(<1$ RPKM). 


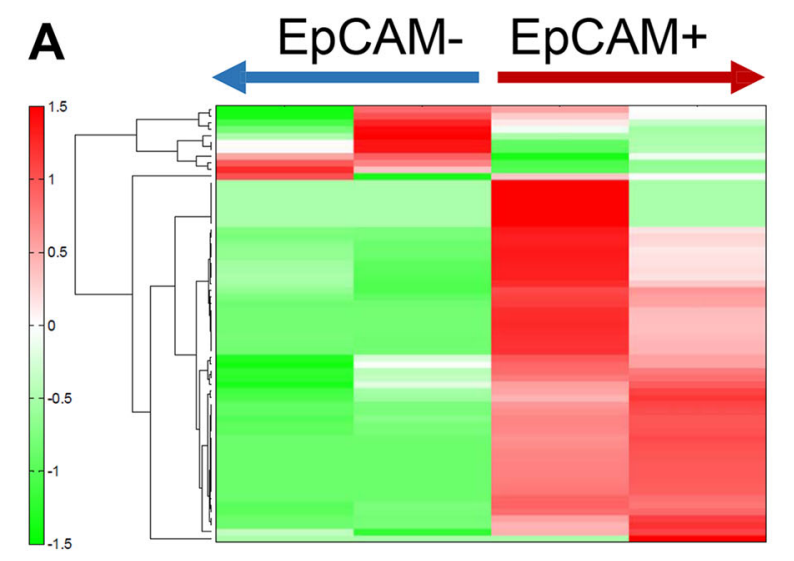

B

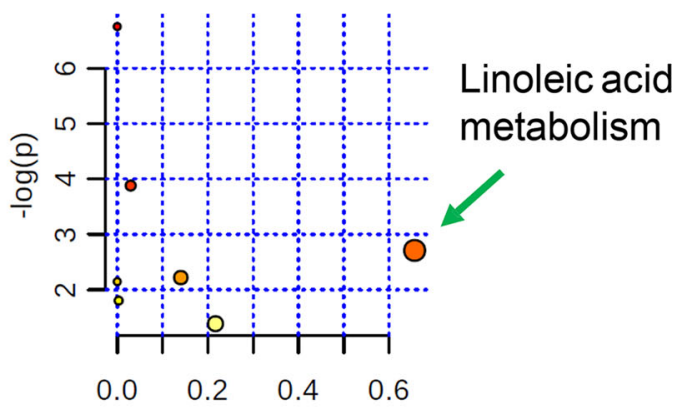

Pathway Impact

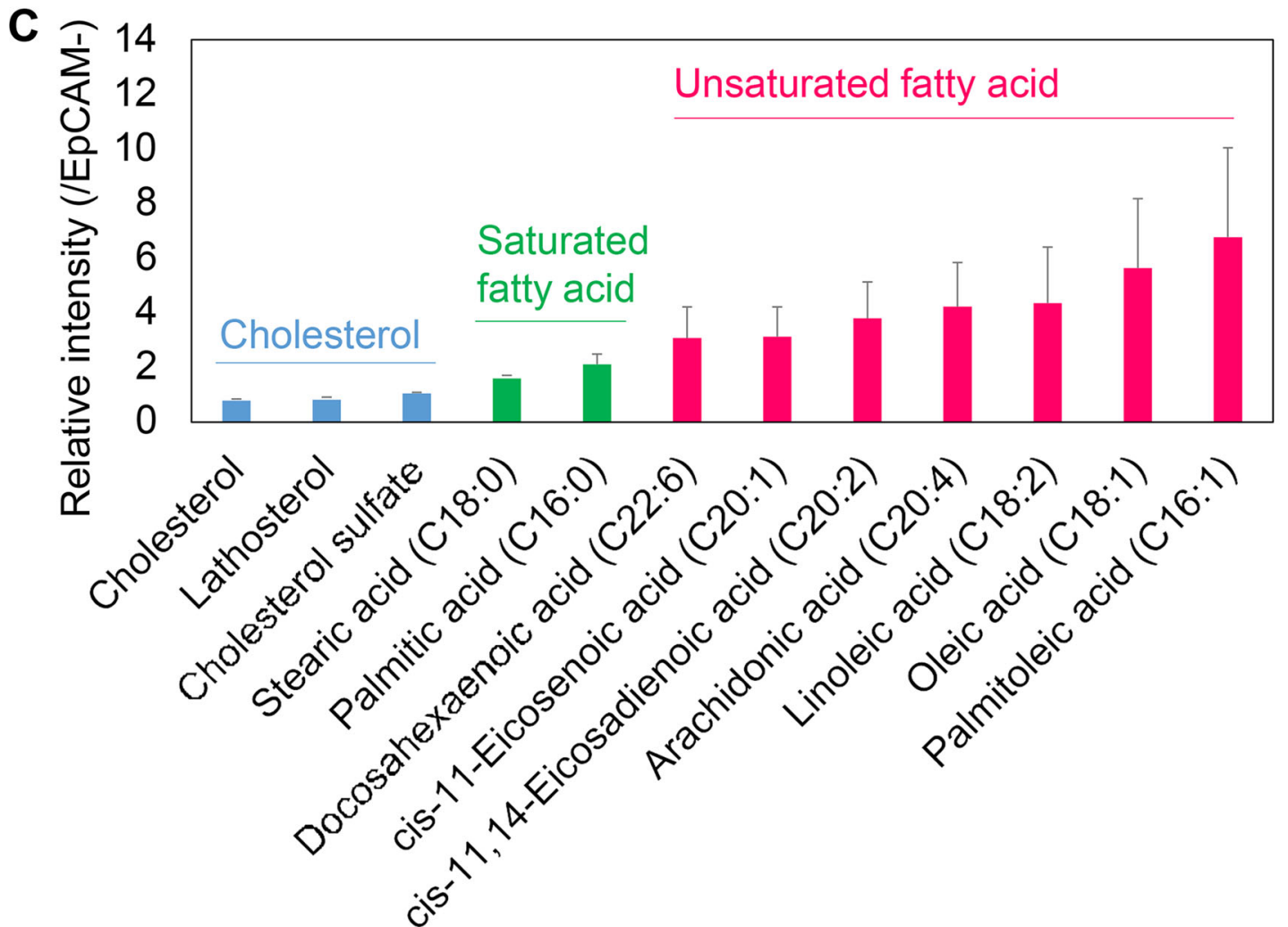

Fig. 1 Metabolome characterization of EpCAM ${ }^{+/-}$HCC cells. The sorted EpCAM ${ }^{+/-} \mathrm{JHH7}$ cells were used. a The clusters generated by hierarchical cluster analysis (HCA) were applied to the lipophilic metabolic profiles detected using a LC-TOFMS-based metabolomics technique. $\mathbf{b}$ The

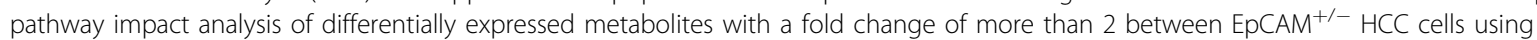

MetaboAnalyst 4.0. Metabolic pathways with values $>0.1$ were considered to be perturbed. c Relative intensity of cholesterol, saturated fatty acids, and unsaturated fatty acids. The data are presented as the fold changes between $\mathrm{EpCAM}^{+/-} \mathrm{JHH}$ cells.

Hierarchical clustering demonstrated enrichment in $\mathrm{MYCN}^{\text {high }} \mathrm{HCC}$ cells for genes related with liver CSC markers (EpCAM and $A F P)$, stemness regulation (DLK1, $N A N O G$, and $A L D H 1 A 1)$, and fatty acid metabolism (ACACA, FASN, FADS1, SCD1, and FADS2) (Fig. 2c). In contrast, MYCN ${ }^{\text {low }}$ HCC cells were characterized by the expression of liver CSC marker CD90 (THY1) and genes involved in glucose metabolism (PKM2 and $L D H A)$ and epithelial-to-mesenchymal transition ( $c-K I T$ and TGF $\beta 1)$ (Fig. 2c).

For further functional analysis, HLF and JHH7 cells were used as $\mathrm{MYCN}^{\text {low }}$ and $\mathrm{MYCN}^{\text {high }} \mathrm{HCC}$ cells, respectively. Morphological analysis showed that the HLF cells had features of mesenchymal-like cells, while the 


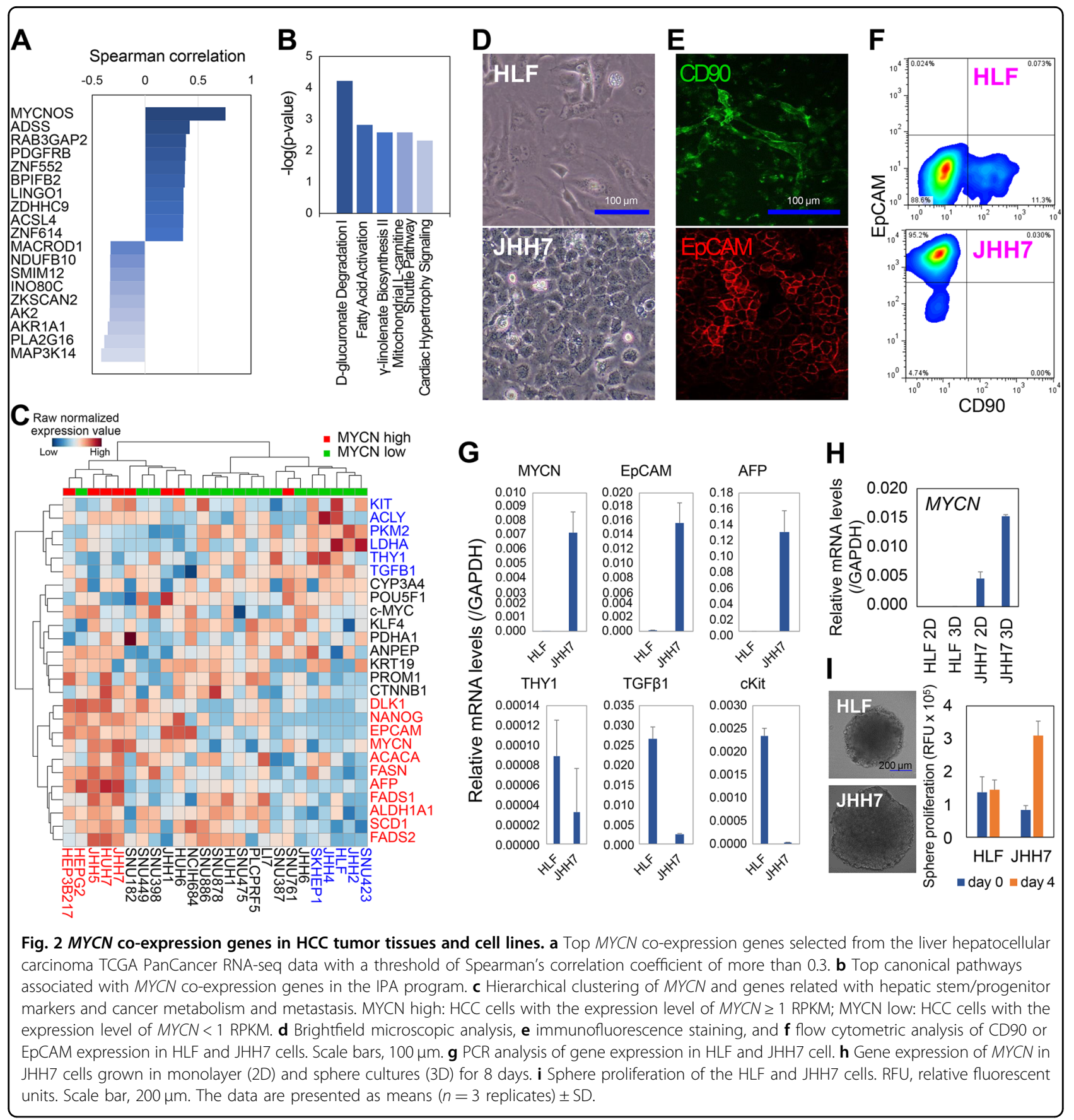

JHH7 cells had those of epithelial cells (Fig. 2d). Immunofluorescence staining (Fig. 2e) and flow cytometric analysis (Fig. 2f) revealed that the HLF cells were negative for EpCAM and positive for CD90, while in contrast JHH7 cells were positive for EpCAM and negative for CD90. PCR analysis confirmed that the gene expression levels of $M Y C N, E p C A M$, and $A F P$ were dramatically higher, while the expression levels of THY1,TGFß1, and $c$-KIT were lower in the JHH7 cells than that in the HLF cells (Fig. 2g). Then, we examined the $M Y C N$ expression in a three-dimensional (3D) multicellular tumor spheroid system, which could mimic in vivo 3D tumor growth and promote stemness maintenance ${ }^{28}$. 3D culture increased the MYCN gene expression in JHH7 cells, but not in HLF cells (Fig. 2h). Furthermore, the JHH7 cells showed greater sphere growth ability than the HLF cells (Fig. 2i).

\section{Loss of function analysis of SCD1 in MYCN ${ }^{\text {high }} \mathrm{HCC}$ cells}

The above data suggested that $M Y C N$ was highly expressed in epithelial-like HCC cells with preferentially 
high tumorigenic potential and CSC characteristics, which was probably related with the acceleration of unsaturated fatty acid synthesis. To examine this hypothesis, we examined whether the inhibition of lipid desaturation using a chemical inhibitor (CAY10566) or shRNA against SCD1 might play a functional role in the proliferation of $\mathrm{MYCN}^{\text {high }} \mathrm{HCC}$ cells in monolayer and sphere cultures. CAY10566 inhibited the spheroid proliferation in the JHH7 cell 3D culture system (Fig. 3a). To provide functional evidence supporting the lipid desaturationmediated effects of CAY10566, we measured sphere proliferation with exogenously added lipid supplementation. The combined addition of monounsaturated fatty acids, $\mathrm{OA}$ and PA, and polyunsaturated fatty acid LA, but not the saturated fatty acid SA, restored the suppression of sphere proliferation by CAY10566 (Fig. 3b). Furthermore, we compared the effect of CAY10566 on the proliferation of MYCN ${ }^{\text {low }}$ HLF cells and $\mathrm{MYCN}^{\text {high }} \mathrm{JHH}$ cells (Figure S1). Notably, a significantly greater growth suppressive effect of CAY10566 was observed in JHH7 cells than in HLF cells when grown as both monolayer and spheres. These data supported SCD1-mediated lipid desaturation as a major player in maintaining the sphere growth of MYCN ${ }^{\text {high }}$ HCC cells. Similarly, knockdown of SCD1 using its shRNA inhibited the proliferation of JHH7 cells in monolayer culture (Fig. 3c) and there was an increased formation of apoptotic bodies (Fig. 3d). Furthermore, flow cytometric analysis demonstrated that the proportion of Annexin $\mathrm{V}$ positive apoptotic cells was increased among these cells (Fig. 3e). PCR analysis also revealed that the expression of pro-apoptotic gene Bcl-2like protein 11 (BCL2L11; commonly called Bim) was increased following SCD1 knockdown in JHH7 cells in both monolayer and sphere cultures (Fig. 3f). Moreover, knockdown of $S C D 1$ led to the decrease in $M Y C N$ gene expression in $\mathrm{JHH} 7$ cells, suggesting that SCD1-mediated signaling pathway might act as an upstream regulator of MYCN gene expression in HCC cells.

\section{RNA-seq based transcriptome analysis identifies molecular} targets of SCD1 in MYCN ${ }^{\text {high }}$ HCC cells

To explore the mechanism underlying the regulation of MYCN gene expression by SCD1, RNA-sequencing (RNA-seq) based transcriptome analysis was performed to identify the molecular targets of SCD1 in JHH7 cells with transient transfection of a control siRNA ( $\mathrm{siCtl}$ ) or siRNAs against SCD1 (siSCD1) or MYCN (siMYCN) (Fig. 4a), and FACS-sorted $\mathrm{CD}^{+/-}$HLF cells and EpCAM $^{+/-}$JHH7 cells (Fig. 4b). Hierarchical clustering of the RNA-seq based transcriptome profiles with 16,735 genes demonstrated diverse expression profiles between HLF and JHH7 cells (Fig. 4c and Table S2). In accordance with the above PCR analysis, the $M Y C N$ gene expression was not detected in $\mathrm{CD} 90^{+/-}$HLF cells, while it was highly expressed in the $\mathrm{EpCAM}^{+} \mathrm{JHH} 7$ cells as compared with that in the $\mathrm{EpCAM}^{-}$cells and was decreased following knockdown of SCD1 or MYCN in JHH7 cells (Fig. 4d). Furthermore, $M Y C N$ gene expression was not correlated with that of $c-M Y C$, which is a major member of the MYC family contributing to the genesis of human cancers ${ }^{16}$ (Fig. 4d). With a threshold fold change of more than 4, differentially expressed genes between $\mathrm{CD} 90^{+/-}$ HLF cells (383 genes), EpCAM ${ }^{+/-}$JHH7 cells (524 genes), and siSCD1/siCtl (284 genes) and siMYCN/siCtl (228 genes) JHH7 cells were selected for pathway analysis to gain greater insights into the biological function (Fig. 4e). Top diseases or functional annotation (Fig. 4f) and upstream regulator analysis (Fig. 4g) using IPA platform revealed that $\mathrm{EpCAM}^{+} \mathrm{JHH} 7$ cells and $\mathrm{CD}^{+}{ }^{+}$HLF cells shared similar activation patterns, such as activation of DNA metabolism and nuclear protein 1 (Nupr1)-regulated signaling pathways, and inhibition in cell death program and estrogen-related signaling pathways. This data was in agreement with the previous reports that the stemness could be considered as a state acquired but not defined by a distinct cell type or a cell biomarker ${ }^{29}$. Notably, the CSC-related signaling pathways were reversibly affected by knockdown of either SCD1 or MYCN, suggesting that SCD1 and/or MYCN might serve as potential CSC therapeutic targets. To identify the upstream regulators of $M Y C N$ gene expression under the control of SCD1, a total of 152 genes were further selected with a threshold fold change of more than 2 according to the following strategy: (1) differentially expressed by siSCD1 transfection in JHH7 cells; (2) differentially expressed between $\mathrm{EpCAM}^{+/-} \mathrm{JHH} 7$ cells and reversibly changed in comparison to the siSCD1 transfected JHH7 cells; (3) no changes between the CD90 ${ }^{+/-}$HLF cells and by siMYCN transfection in JHH7 cells. Canonical pathways analysis of these genes revealed that endocannabinoid signaling pathways were under the control of SCD1 in HCC cells (Fig. 4h). The most highly populated network was the endocannabinoid cancer inhibition pathway, which contains genes that play critical roles in controlling endoplasmic reticulum (ER) stress such as cyclic AMP response element-binding protein $(C R E B)$, DNA damage inducible transcript 3 (DDIT3), and cyclic AMPdependent transcription factor (ATF3) (Fig. 4i). These data suggested that the $S C D 1$ knockdown-induced ER stress might play a functional role in regulation of $M Y C N$ gene expression in the HCC cells.

\section{ER stress regulates MYCN gene expression in $M Y C N^{\text {high }}$ HCC cells}

The gene expression of $A T F 3$ was examined using PCR. Knockdown of $S C D 1$ induced the significant expression of ATF3 in JHH7 cells (Fig. 5a). In contrast, the gene expression of $A T F 3$ was decreased in the $\mathrm{EpCAM}^{+}$CSC- 

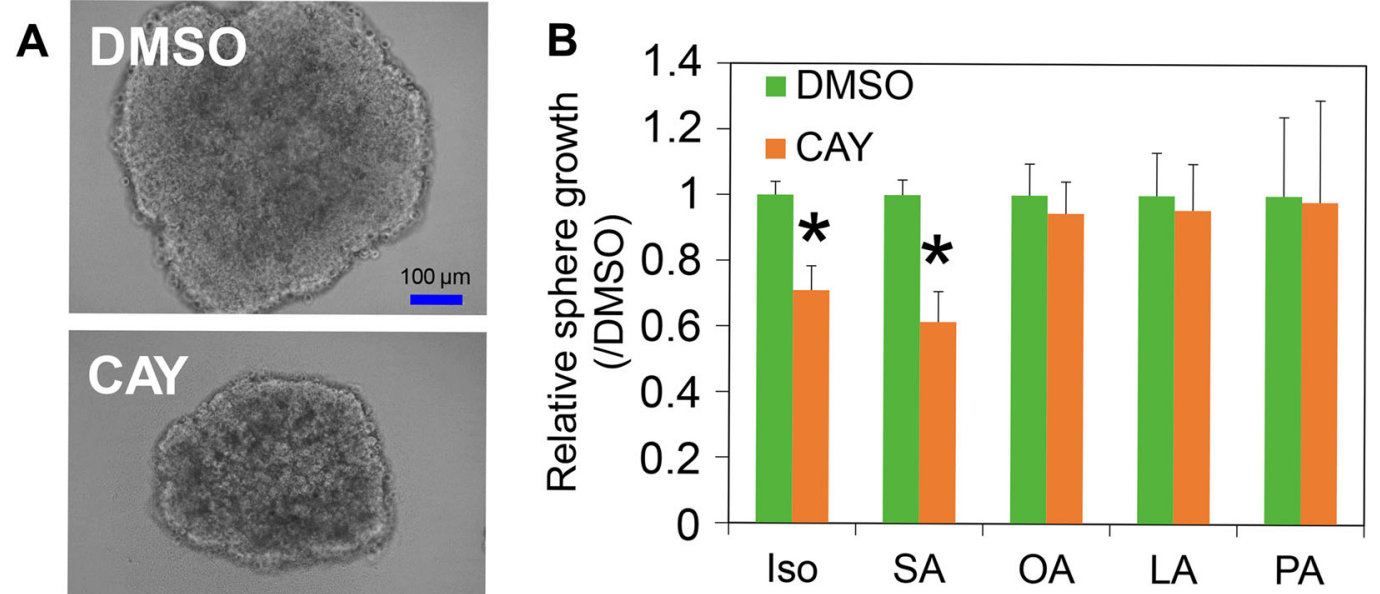

C
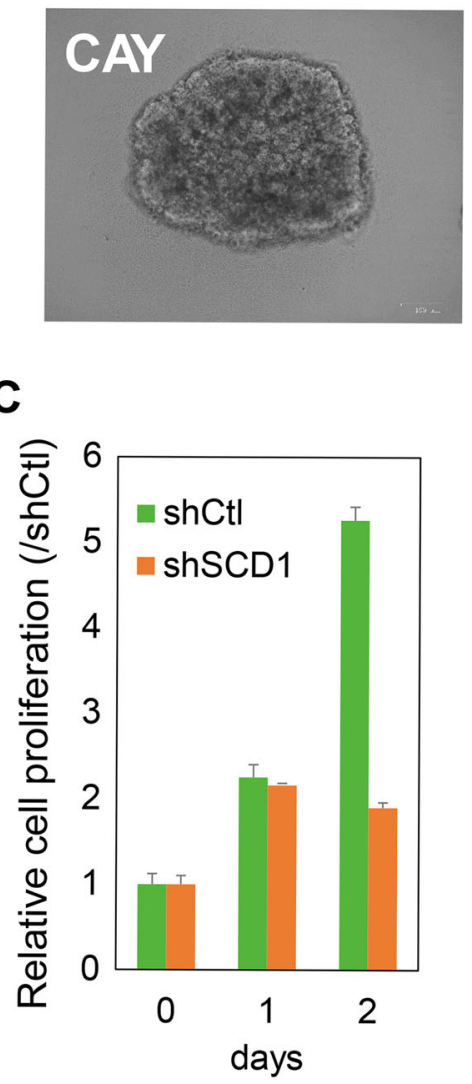

F

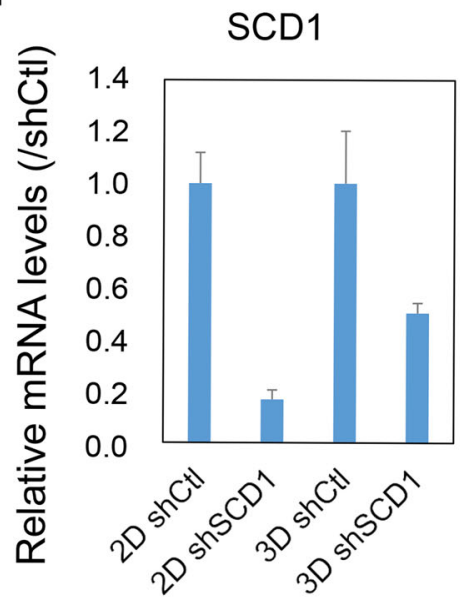

D

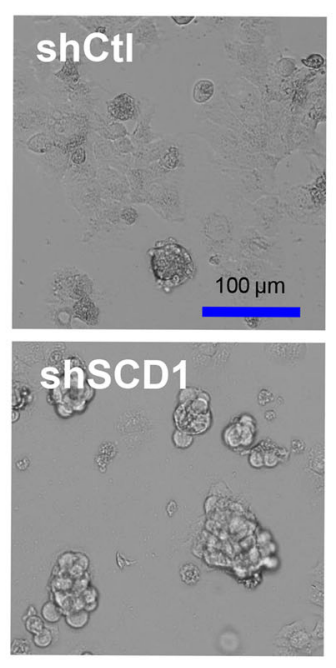

MYCN

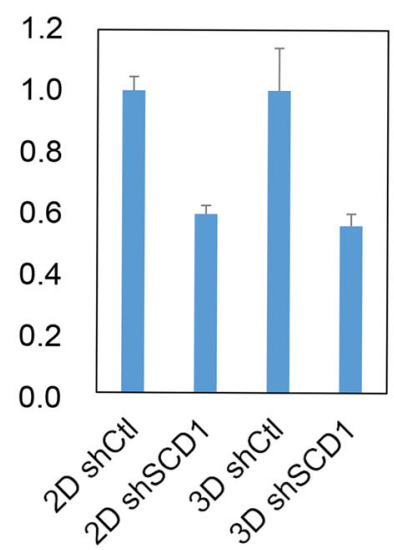

E

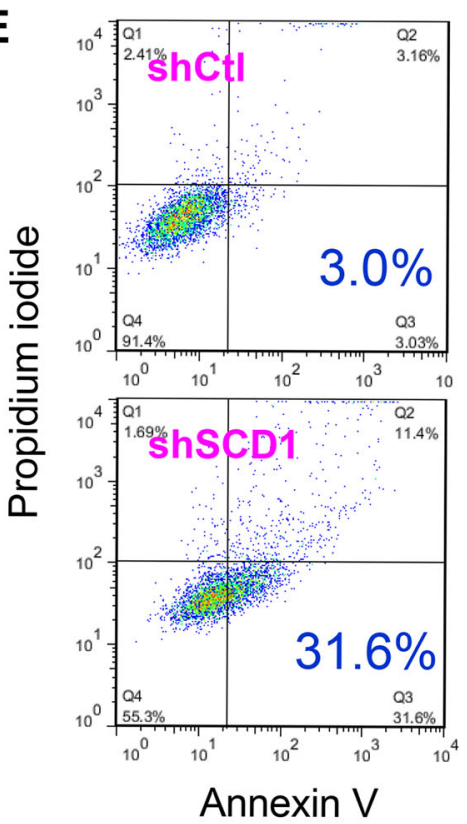

Bim

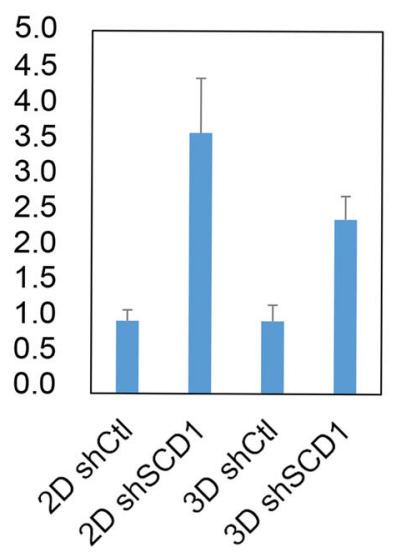

Fig. 3 (See legend on next page.) 
(see figure on previous page)

Fig. 3 Loss of function analysis of SCD1 in MYCN ${ }^{\text {high }}$ HCC cells. a Representative microscopic images of JHH7 cells grown in sphere cultures. The spheres were treated with DMSO or a chemical inhibitor of SCD1, CAY10566 (CAY), at $10 \mu \mathrm{M}$ for 4 days. Scale bar, $100 \mu \mathrm{m}$. b Sphere proliferation of $\mathrm{JHH7}$ cells treated with DMSO or $10 \mu \mathrm{M}$ CAY in the presence or absence of $100 \mu \mathrm{M}$ fatty acids (stearic acid, SA; oleic acid, OA; linoleic acid, LA; palmitoleic acid, PA) for 7 days. Isopropanol (iso) was used as the primary solvent for the fatty acids. c Cell proliferation of control shRNA (shCtl) or shSCD1-transduced JHH7 cells grown in monolayer cultures. $\mathbf{d}$ Representative microscopic images of shCtl or shSCD1-transduced JHH7 cells grown in monolayer cultures for 3 days. Scale bar, $100 \mu \mathrm{m}$. e Apoptotic cell death of shCtl or shSCD1-transduced JHH7 cells grown in monolayer cultures for 3 days was detected by dual staining with Annexin V-FITC and propidium iodide (PI) followed by flow cytometric analysis. f Gene expression of SCD1, MYCN, and BCL2L11 (Bim) in shCtl and shSCD1-transduced JHH7 cells in monolayer (2D) and sphere cultures (3D) for 7 days. The data are presented as means $\left(n=3\right.$ replicates) $\pm S D ;{ }^{*} p<0.05$, Student's $t$-test.

like JHH7 cells than that in the EpCAM ${ }^{-} \mathrm{JHH} 7$ cells (Fig. 5b). The gene expression of ATF3 was also decreased in JHH7 cells grown in sphere cultures than that in monolayer cultures (Fig. 5c). Data mining in the TCGA database revealed a significant negative correlation between the gene expression of $M Y C N$ and $A T F 3$ in human HCC tissues (Fig. 5d). Since ATF3 is an ER stressinducible transcriptional repressor ${ }^{30}$, we then examined whether ER stress might functionally regulate $M Y C N$ gene expression. Tunicamycin, a chemical inducer of ER stress, strongly stimulated the gene expression of $A T F 3$ and inhibited the gene expression of $M Y C N$ in the JHH7 cells (Fig. 5e). Finally, we examined whether ER stress is associated with the suppression of $M Y C N$ gene expression by ACR. Lipid profiling using ${ }^{1} \mathrm{H}$ nuclear magnetic resonance (NMR) spectroscopy revealed that the treatment with ACR for $4 \mathrm{~h}$ dramatically reduced the content of unsaturated fatty acids in JHH7 cells (Fig. 5f). To further explore the molecular basis of the suppressive effect of ACR on lipid desaturation, the effect of ACR on gene expression of lipid desaturases was examined in JHH7 cells (Figure S2). Treatment with ACR for $4 \mathrm{~h}$ significantly suppressed the gene expression of $S C D 1$ as well as fatty acid desaturase-1 (FADS1) in JHH7 cells. ACR significantly enhanced the gene expression of ATF3 in JHH7 cells, while co-addition of tauroursodeoxycholic acid (TUDCA), a chemical inhibitor of ER stress (Fig. 5g), as well as knockdown of ATF3 using siRNA (Fig. 5h) significantly diminished the effects of ACR on ATF3 and MYCN gene expression in JHH7 cells. These data suggested ACR suppressed $M Y C N$ gene expression in JHH7 cells, at least in part through ER stress induced ATF3 signaling pathways.

\section{Discussion}

We have previously reported that (1) inhibition of biosynthesis of unsaturated fatty acids, but not glucose metabolism plays a crucial role in the prevention of hepatocellular carcinogen diethylnitrosamine (DEN)induced hepatic tumorigenesis by ACR in the obesity mice model ${ }^{31}$; (2) ACR can inhibit MYCN gene expression and selectively kill MYCN ${ }^{\text {high }}$ EpCAM $^{+}$CSC-like HCCs, thus, providing a molecular basis by which ACR prevents $\mathrm{HCC}$ recurrence ${ }^{20}$; (3) proteome analysis has shown that $\mathrm{MYCN}^{\text {high }} \mathrm{EpCAM}^{+}$CSC-like $\mathrm{HCC}$ cells are enriched in enzymes involved in lipid desaturation such as SCD1, FADS1 and FADS2 ${ }^{22}$. It should be noted that it is the upregulation of $M Y C N$ mRNA expression, but not its amplification status that is correlated with the prognosis of $\mathrm{HCC}^{20}$. Therefore, it seems that $M Y C N$ gene expression might represent a dynamic stem state of the liver CSC-like cells and an understanding of how $M Y C N$ transcription is activated would provide new insights on liver tumorigenesis. In this study, we further provided evidence that (1) the content of unsaturated fatty acids was increased in $\mathrm{MYCN}^{\text {high }} \mathrm{EpCAM}^{+}$CSC-like HCC cells; (2) inhibition of lipid desaturation using either the chemical inhibitor or siRNA/shRNA against SCD1 suppressed cell proliferation as well as $M Y C N$ gene expression in the $\mathrm{MYCN}^{\text {high }} \mathrm{HCC}$ cells grown as both monolayer and spheres. These data bridge the gap in the knowledge about the link between lipid desaturation and MYCN gene expression in HCC cells.

It is known that MYC family oncogenes activate the expression of the enzymes involved in fatty acid biosynthesis and promote lipid metabolic reprogramming in support of reprogrammed cell growth and survival in tumorigenesis ${ }^{32}$. Blocking unsaturated fatty acid synthesis with chemical inhibitors had been shown to be toxic to Myc-overexpressing cells ${ }^{10}$. In contrast, our study showed a regulatory role of SCD1 on the $M Y C N$ gene expression in HCC cells, suggesting that, in addition to its potential as the major source of energy and building blocks of membranes for cell proliferation, the lipid-rich microenvironment might also contribute to tumorigenesis via mediating oncogenic signaling. Obesity might cause chronic inflammation via promoting the production of tumor-promoting cytokines such as IL-6 and activation of oncogenes such as STAT3, which has a critical role in regulating cell fate, inflammation, and immunity ${ }^{33}$. A new study has demonstrated that the fatty acids could directly activate STAT3 by enhancing its palmitoylation ${ }^{34}$. In addition, there is increasing focus on the emerging roles of lipid metabolism, especially SCD1-mediated lipid desaturation, in the generation and maintenance of $\mathrm{CSCs}$, through the activation of oncogenic signaling pathways such as NF- $\mathrm{kB}, \mathrm{Wnt} / \beta$-catenin and Hippo/YAP 


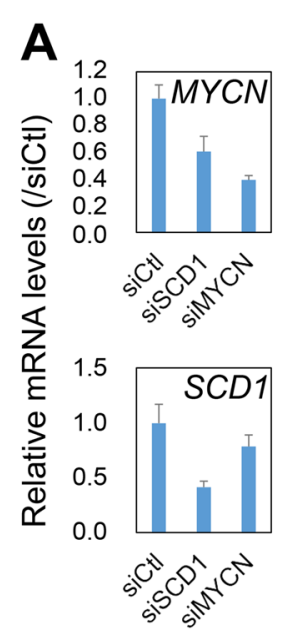

B

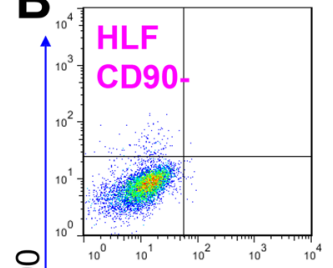

ํ.

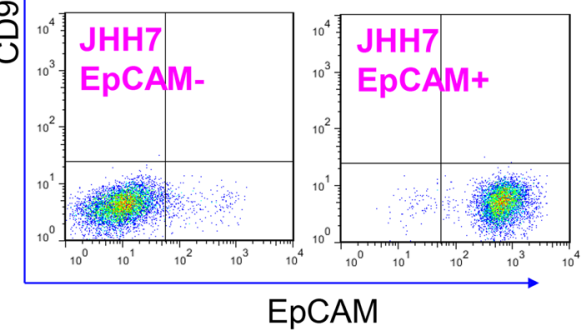

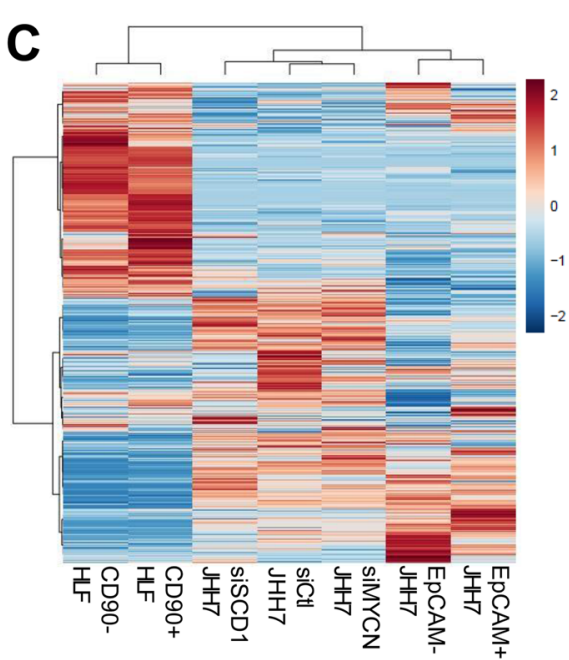

G
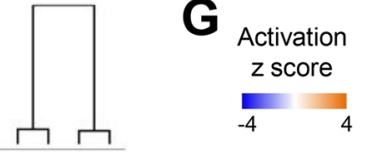

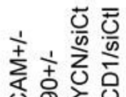

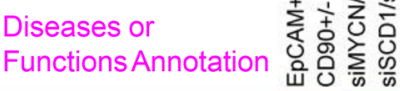

Metabolism of DNA

Quantity of cells

Size of body

Extracranial solid tumor

Cancer

Cell death of brain

Incidence of tumor

Malignant solid tumor

Incidence of lymphoma

Sensitivity of cells

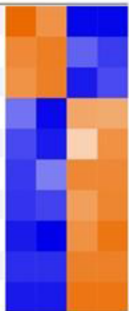

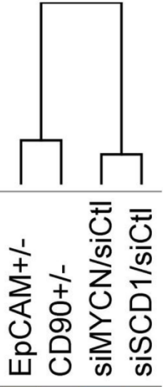
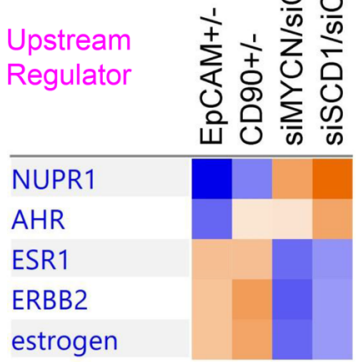

$\mathrm{H}$ -log(p-value)

\section{Endocannabinoid} Cancer Inhibition Pathway

\section{Role of BRCA1 in DNA} Damage Response

\section{Apelin Pancreas Signaling Pathway \\ Endocannabinoid Developing Neuron Pathway \\ Apelin Adipocyte Signaling Pathway}

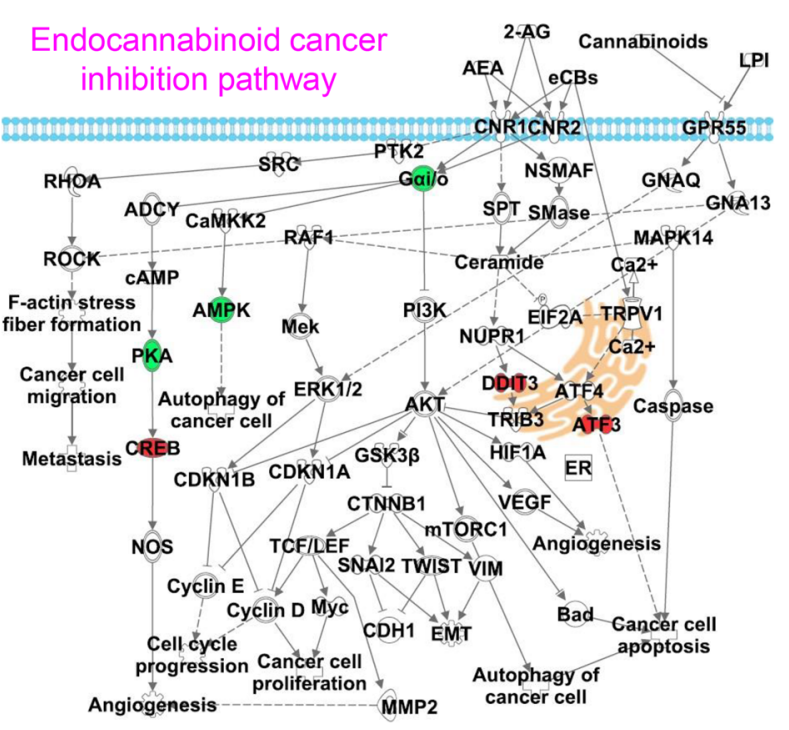

Fig. 4 (See legend on next page.) 


\begin{abstract}
(see figure on previous page)
Fig. 4 RNA-seq based transcriptome analysis identifies molecular targets of SCD1 in MYCN ${ }^{\text {high }}$ HCC cells. a MYCN (upper) and SCD1 (lower) gene expression in $\mathrm{JHH} 7$ cells treated with $100 \mathrm{nM}$ control siRNA (siCtl), siSCD1 or siMYCN for $24 \mathrm{~h}$. b Double-staining flow cytometric analysis of CD90 and EpCAM in FACS-isolated CD90 ${ }^{+/-}$HLF cells (upper) and EpCAM ${ }^{+/-}$JHH7 cells (lower). c Hierarchical clustering of RNA-seq based transcriptome profiles of $\mathrm{CD} 0^{+-}$HLF cells, EpCAM ${ }^{+-}$JHH7 cells and JHH7 cells treated with $100 \mathrm{nM}$ siCtl, siSCD1 or siMYCN for $24 \mathrm{~h}$. d Expression of MYCN and C-MYC measured by RNA-seq analysis. FPKM, fragments per kilobase of exon model per million reads mapped. e Numbers of differentially expressed genes with a threshold of change of more than 4-fold. $\mathbf{f}$ Top disease or functional annotation and $\mathbf{g}$ upstream regulator associated with differentially expressed genes between $\mathrm{EpCAM}^{+/-} \mathrm{JHH} 7$ cells, CD90 ${ }^{+/-} \mathrm{HLF}$ cells, siMYCN/siCtl-treated JHH7 cells, and siSCD1/siCtltreated JHH7 cells performed in IPA platform. The pathways were ranked according to their activation z score, which can be used to predict either activated or inhibited state based on a statistically significant pattern match of up- and downregulation, respectively. $\mathbf{h}$ Top highly populated canonical pathways associated with a total of 154 genes (1) differentially expressed by siSCD1 transfection in JHH7 cells; (2) differentially expressed between $\mathrm{EPCAM}^{+-} \mathrm{JHH7}$ cells and reversibly changed in compared with siSCD1 transfected JHH7 cells; (3) with no changes between $\mathrm{CD}^{+1} 0^{+/-} \mathrm{HLF}$ cells and by siMYCN transfection in JHH7 cells. i A representative network under control of SCD1 in JHH7 cells called the endocannabinoid cancer inhibition pathway. Upregulated genes are indicated in red, downregulated genes indicated in green, and genes that were not annotated in this study, but are part of this network were indicated in white.
\end{abstract}

signaling $^{35}$. For example, monounsaturated fatty acids such as OA might support mRNA stabilization of the Wnt receptor LRP5/6 and contribute to the activation of Wnt/ $\beta$-catenin signaling, which is critical for maintaining stem cell pluripotency ${ }^{36}$. Therefore, targeting lipid desaturation should be a promising therapeutic strategy for HCC treatment via the inhibition of energy production and membrane building blocks, as well as for HCC prevention through the suppression of oncogenic signaling and elimination of CSCs.

We further showed through transcriptome analysis that the ER stress related signaling networks such as endocannabinoid cancer inhibition pathway were under the control of SCD1 in MYCN ${ }^{\text {high }}$ HCC cells. Furthermore, the chemical inducer of ER stress inhibited $M Y C N$ gene expression, while the chemical inhibitor of ER stress partially rescued the suppression of $M Y C N$ gene expression by ACR in MYCN ${ }^{\text {high }}$ HCC cells. Lipid messengers, such as sphingolipids, lysolipids, and endocannabinoids, play critical roles in multiple cellular functions from cell proliferation to apoptosis and are emerging as promising therapeutic targets in cancer ${ }^{37}$. Endocannabinoids are endogenous lipid-based retrograde neurotransmitters and their G-protein-coupled receptors $\mathrm{CB} 1$ and $\mathrm{CB} 2$ are expressed throughout the nervous system. CB1 activation causes the generation of the lipid second messenger ceramide, which plays central roles in cannabinoid induced apoptosis, partly through triggering of ER stress pathways ${ }^{38}$. The $M Y C N$ oncogene is notably amplified in peripheral and central nervous system tumors especially neuroblastoma ${ }^{18}$. In addition, retinoic acids have been shown to transcriptionally induce $C B 1$ expression in hepatocytes via retinoic acid receptor $\gamma$-dependent pathways ${ }^{39}$. Therefore, it is possible that ACR regulates MYCN gene expression through endocannabinoid system-mediated ER stress signaling pathways.

It has been reported that decrease in membrane phospholipid unsaturation and increase in cellular saturated fatty acids induced ER stress-dependent cell death ${ }^{40,41}$. Indeed, activation of ER stress signaling is instrumental in the development of NASH-related HCC by triggering chronic liver damage and inflammation ${ }^{7}$. In contrast, tissue stem cells, as well as CSCs, are featured with their adaptive plasticity to allow them to survive when exposed to a wide range of stresses including ER stress ${ }^{42}$. Reduction in ER stress enables maintenance of functional hematopoietic stem cells ${ }^{43}$, while induction of ER stress causes loss of intestinal epithelial stemness ${ }^{44}$. Importantly, rescue of ER stress in CSC induces differentiation and sensitizes them to chemotherapy in colon cancer ${ }^{45}$ and $\mathrm{HCC}^{46}$. In agreement with this, we found that the expression of ER stress-inducible gene ATF3 was downregulated in $\mathrm{MYCN}^{\text {high }} \mathrm{EpCAM}^{+} \mathrm{CSC}$-like $\mathrm{HCC}$ cells and CSC-rich spheroids, which was upregulated by ACR treatment or lipid desaturation inhibition. Therefore, escaping from the ER stress-mediated programmed cell death in obesity-caused inflammatory microenvironment should play a critical role in the maintenance of liver CSCs and hepatic tumorigenesis and serve as a promising therapeutic target in the prevention of HCC.

In summary, multi-omics analyses revealed that lipid desaturation-mediated ER stress signaling reversibly regulates the MYCN gene expression in HCC cells and might be a promising therapeutic target for the treatment and prevention of HCC.

\section{Materials and methods \\ Cell culture}

The HCC cell line HLF (JCRB0405) was obtained from the Japanese Collection of Research Bioresources Cell Bank ${ }^{47}$. The HCC cell line JHH7 was kindly provided by Prof. T. Matsuura of the Jikei University School of Medicine, Tokyo, Japan ${ }^{48}$. The cells were maintained at $37^{\circ} \mathrm{C}$ and $5 \% \mathrm{CO}_{2}$, in Dulbecco's modified Eagle medium (DMEM, Wako Industries, Osaka, Japan) containing 10\% fetal bovine serum (FBS; Mediatech, Herndon, VA, USA), $100 \mathrm{U} / \mathrm{mL}$ penicillin/ streptomycin, and $2 \mathrm{mM}$ L-glutamine (Mediatech). 


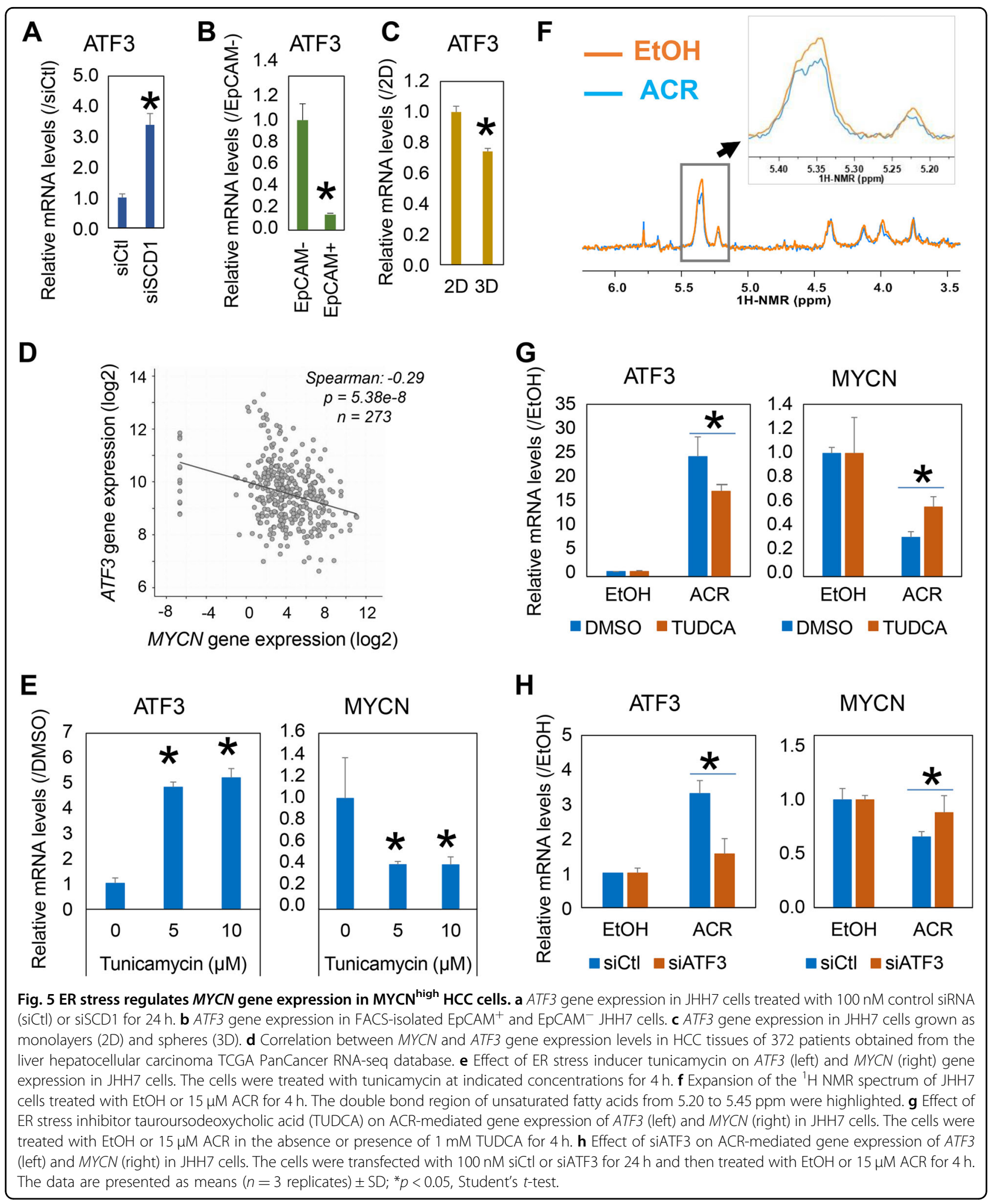

\section{Chemicals}

A SCD1 inhibitor, CAY10566 (ab144421), was purchased from Abcam (Cambridge, MA, USA). Tunicamycin (11445) and TUDCA (20277) were purchased from
Cayman Chemical (Ann Arbor, MI, USA). Fatty acids SA (S4751), OA (O1008), PA (P0500), and LA (L1376) were obtained from Sigma (Louis, MO, USA). ACR was supplied by Kowa Co. Ltd (Tokyo, Japan). Dimethyl sulfoxide 
(DMSO, Sigma) was used as the primary solvent for CAY10566, tunicamycin, and TUDCA. Isopropanol (Wako Industries) was used as the primary solvent for fatty acids. Ethanol (EtOH, Wako Industries) was used as the primary solvent for ACR.

\section{FACS sorting}

Flow cytometric analysis was performed as previously described $^{20}$. The cells were incubated with Alexa Fluor 647-conjugated mouse anti-EpCAM (1:20; 324212; BioLegend, San Diego, CA, USA) and/or FITC-conjugated mouse anti-CD90 (328108; BioLegend) at $4{ }^{\circ} \mathrm{C}$ for $30 \mathrm{~min}$. The labeled cells were analyzed and sorted on a $\mathrm{BD}$ FACSAria (BD Biosciences, San Diego, CA, USA). The data were analyzed in FlowJo software (Tree Star, Inc., Ashland, OR, USA).

\section{LC-TOFMS metabolome analysis}

The cells were washed with $5 \%(\mathrm{w} / \mathrm{w})$ mannitol solution twice and harvested in $\mathrm{EtOH}$ containing $10 \mu \mathrm{M}$ internal standards (Human Metabolome Technologies Inc., Tsuruoka, Japan) with scrapers. The cell pellet was diluted in $1000 \mu \mathrm{L}$ Milli-Q water and supernatant was collected by centrifugation $\left(4400 \times g, 4{ }^{\circ} \mathrm{C}, 5 \mathrm{~min}\right)$. The supernatant was dried and resuspended in $200 \mu \mathrm{L}$ of isopropanol/ Milli-Q water solution (1:1) for LC-TOFMS analysis using an Agilent 1200 series RRLC system SL equipped with an Agilent LC/MSD TOF system (Agilent Technologies, Palo Alto, CA, USA) ${ }^{31}$.

\section{D spheroid cultures}

3D spheroid cultures were performed on non-adherent 96-well round-bottomed Sumilon PrimeSurface ${ }^{\mathrm{TM}}$ plates (MS-9096U, Sumitomo Bakelite, Tokyo, Japan) as previously described ${ }^{28}$. The spheroids were grown for 4 days, then $50 \mu \mathrm{L}$ of media was replaced with $50 \mu \mathrm{L}$ fresh serumfree media containing $10 \mu \mathrm{M}$ CAY10566, and/or $100 \mu \mathrm{M}$ fatty acids. The spheroids were further cultured for 7 days and photos were taken using an optical microscope (DSFi1, NIKON, Tokyo, Japan).

\section{Spheroid proliferation assay}

Spheroid proliferation was measured using the CellTiter-Fluor $^{\mathrm{TM}}$ Cell Viability Assay (Promega Corporation, Madison, WI, USA). The fluorescence was measured using a plate reader ( $\lambda$ excitation/emission: $390 \mathrm{~nm} /$ $505 \mathrm{~nm}$ ) (ARVO MX, Perkin Elmer Inc., Waltham, MA, USA).

\section{In vitro RNA interference}

A pool of 3 target-specific siRNAs targeting human SCD1 (sc-36464; siSCD1), human MYCN (sc-36003; siMYCN), human ATF3 (sc-44283; siATF3) and a control siRNA (sc-37007; siCtl) were purchased from Santa Cruz
Biotechnology (Santa Cruz, CA, USA). The cells were plated in 24-well plates for $24 \mathrm{~h}$, and then transfected with $100 \mathrm{nM}$ siRNAs using lipofectamine 2000 transfection reagent (Life Technologies, Gaithersburg, MD, USA). On the following day, cells were collected for RNA isolation or treated with chemicals for further analysis.

\section{Transduction of shRNA lentiviral particles}

SCD1 (sc-36464-V) and control (sc-108080) short hairpin RNA (shRNA) lentiviral particles were obtained from Santa Cruz Biotechnology. The cells were transduced with lentiviral vectors expressing the shRNAs at $\sim 0.5$ multiplicity of infection (MOI) using $5 \mu \mathrm{g} / \mathrm{mL}$ Polybrene (Santa Cruz Biotechnology) and then selected with $2 \mu \mathrm{g} / \mathrm{mL}$ puromycin-containing culture medium.

\section{Immunofluorescence analysis}

The cells were seeded in 96-well plates at $10^{5}$ cells $/ \mathrm{mL}$. After $24 \mathrm{~h}$, the cells were stained with Alexa Fluor 647conjugated mouse anti-EpCAM $(1: 20 ; 324212$; BioLegend) and/or FITC-conjugated mouse anti-CD90 (1:20; 328108; BioLegend) at room temperature for $30 \mathrm{~min}$. Images were captured using an ImageXpress Micro Confocal High-Content Imaging System (Molecular Devices, Sunnyvale, CA, USA).

\section{RNA isolation and real-time (RT)-PCR}

Total RNA was isolated using a FastGene RNA Basic Kit (FG-80250, NIPPON Genetics, Tokyo, Japan) and quantified using a NanoDrop spectrophotometer (NanoDrop products, Wilmington, DE, USA). cDNA was synthesized using a PrimeScript RT Master Mix Kit (TaKaRa Bio, Otsu, Japan). Primer sequences were as follows: glyceraldehyde 3-phosphate dehydrogenase $(G A P D H)$ forward (5' CAATGACCCCTTCATTGACC $\left.3^{\prime}\right)$ and reverse $\left(5^{\prime}\right.$ GACAAGCTTCCCGTTCTCAG 3'), SCD1 forward (5' GTACCGCTGGCACATCAACTT $3^{\prime}$ ) and reverse (5' TTGGAGACTTTCTTCCGGTCAT 3'), FADS1 forward (5' CTACCCCGCGCTACTTCAC $3^{\prime}$ ) and reverse $\left(5^{\prime}\right.$ CGGTCGATCACTAGCCACC $3^{\prime}$ ), MYCN forward (5' GGCAGTAGGACCACCAGTGT $3^{\prime}$ ) and reverse $\left(5^{\prime}\right.$ AACCGTCACCAACGTTTAGC 3'), BCL2L11 forward (5' GGCCCCTACCTCCCTACA $3^{\prime}$ ) and reverse $\left(5^{\prime}\right.$ GGGGTTTGTGTTGATTTGTCA $3^{\prime}$ ), and ATF3 forward (5' TTTGCTAACCTGACGCCCTT $3^{\prime}$ ) and reverse (5' TGACTGATTCCAGCGCAGAG $3^{\prime}$ ). PCR reactions were performed using a Roche LightCycler 96 Real-Time PCR System (Roche Diagnostic Co., Ltd., Tokyo, Japan) and the SYBR Premix ExTaq II (TaKaRa Bio).

\section{Data mining}

RNA-seq transcriptome profiles of $372 \mathrm{HCC}$ tumor tissues (PanCancer Atlas) were obtained from the TCGA database using cBioPortal software (www.cbioportal.org/) ${ }^{49}$. 
RNA-seq transcriptome profiles of $25 \mathrm{HCC}$ cell lines were obtained from the CCLE database (https://portals. broadinstitute.org/ccle) $)^{27}$.

\section{Cell viability assay}

Cell viability was measured using a Cell Counting Kit-8 (Dojindo Molecular Technologies, Tokyo, Japan). The absorbance was measured using a plate reader (ARVO MX, Perkin Elmer Inc.) at $450 \mathrm{~nm}$.

\section{Annexin V staining assay}

Apoptotic cells were detected using the FITC Annexin V Apoptosis Detection Kit with Propidium iodide (PI) (640914, BioLegend). Briefly, the cells were washed thoroughly with cell staining buffer (420201, BioLegend) and resuspended in Annexin binding buffer. The cells were then labeled with Annexin V-FITC and PI at room temperature for $15 \mathrm{~min}$ in the dark. The fluorescence of stained cells was measured using a BD LSR flow cytometer and CellQuest Pro software (BD Biosciences). The data were further analyzed in Flowjo software (Tree Star, Inc.).

\section{RNA-seq based transcriptome analysis}

RNA-Seq libraries were prepared using TruSeq Stranded mRNA Library Prep Kit (Illumina, San Diego, CA, USA), followed by paired-end sequencing on a NovaSeq 6000 instrument (Illumina). STAR (2.5.1b) was used to align reads in fastq files to the UCSC human hg38 reference genome. Stringtie (1.3.5) was used to assemble the transcriptome based on the hg38 reference annotation with Gencode.v30. The quantification of relative abundance of each transcript was reported as fragments per kilobase of exon model per million reads mapped (FPKM). Data downloads are available (Table S2).

\section{Knowledge-based pathway analysis}

For the metabolome analysis, the pathway impact was calculated as the sum of the importance measures of the matched metabolites normalized by the sum of the importance measures of all metabolites in each pathway using MetaboAnalyst $4.0^{50}$. For the transcriptome analysis, the knowledge-based functional analysis was performed using the IPA platform (Ingenuity Systems, Mountain View, CA, USA) as previously described ${ }^{19}$. The generated biological networks were ranked by score, which is the likelihood of a set of genes being found in the network owing to random chance, identified by a Fisher's exact test. The generated diseases or functional annotations or upstream regulators were ranked by the activation $\mathrm{z}$ score, which can be used to find likely regulating molecules based on a statistically significant pattern match of up- and downregulation, and also to predict the activation state (either activated or inhibited) of a putative regulator. An absolute $\mathrm{z}$ score of more than 2 was considered as significant.

\section{Lipid profiling using ${ }^{1} \mathrm{H}$ NMR spectroscopy}

The JHH7 cells treated with EtOH or $15 \mu \mathrm{M}$ ACR for $4 \mathrm{~h}$ were harvested and lipid was extracted using the chloroform/methanol/water extraction method as previously described ${ }^{51}$. The ${ }^{1} \mathrm{H}$ NMR spectra were measured at $700 \mathrm{MHz}$ on a Bruker AVANCE II 700 spectrometer (Bruker BioSpin $\mathrm{GmbH}$, Rheinstetten, Germany). All NMR spectra were processed using the MestReNova program (Version 12.0.1, MestRec, Santiago de Compostela, Spain). Tetramethylsilane was used as the internal standard. The double bond regions of unsaturated fatty acids appear mainly from 5.20 to $5.45 \mathrm{ppm}$.

\section{Statistical analysis}

Quantitative data are expressed as the mean \pm SD of at least three replicates. The significance of differences between values was assessed using Student's $t$-test. A $p$ value $<0.05$ was considered significant.

\section{Acknowledgements \\ We thank Dr. Kosuke Hashimoto (RIKEN Center for Integrative Medical Sciences) and Mr. Kenji Ohtawa (RIKEN Research Resources Center) for technical support in RNA-seq analysis and flow cytometric analysis, respectively. This work was supported by grants from the Ministry of Education, Culture, Sports, Science and Technology of Japan's Grant-in-Aid for Young Scientists, JP18K15833 (to X. Y.Q.), a Research on the Innovative Development and the Practical Application of New Drugs for Hepatitis B Grant, JP19fk0310112, from the Japan Agency for Medical Research and Development (to S.K.), and Takeda Science Foundation fund (to X.Y.Q.). T.S. was awarded the International Program Associate (IPA) fellowship from the RIKEN, Japan.}

\section{Conflict of interest}

The authors declare that they have no conflict of interest. The funders had no role in the design of the study; in the collection, analyses, or interpretation of data; in the writing of the paper; in the decision to publish the results.

\section{Publisher's note}

Springer Nature remains neutral with regard to jurisdictional claims in published maps and institutional affiliations.

Supplementary Information accompanies this paper at (https://doi.org/ 10.1038/s41419-020-2257-y).

Received: 2 October 2019 Revised: 11 January 2020 Accepted: 13 January 2020

Published online: 27 January 2020

\section{References}

1. Pikarsky, E. et al. NF-kappaB functions as a tumour promoter in inflammationassociated cancer. Nature 431, 461-466 (2004).

2. Venook, A. P., Papandreou, C., Furuse, J. \& de Guevara, L. L. The incidence and epidemiology of hepatocellular carcinoma: a global and regional perspective. Oncologist 15(Suppl 4), 5-13 (2010)

3. El-Serag, H. B. Epidemiology of viral hepatitis and hepatocellular carcinoma. Gastroenterology 142, 1264-1273 e1261 (2012).

4. Hosaka, T. et al. Long-term entecavir treatment reduces hepatocellular carcinoma incidence in patients with hepatitis B virus infection. Hepatology $\mathbf{5 8}$ 98-107 (2013). 
5. Singal, A. K. et al. Antiviral therapy reduces risk of hepatocellular carcinoma in patients with hepatitis C virus-related cirrhosis. Clin. Gastroenterol. Hepatol. 8 192-199 (2010)

6. Calle, E. E., Rodriguez, C., Walker-Thurmond, K. \& Thun, M. J. Overweight, obesity, and mortality from cancer in a prospectively studied cohort of U.S. adults. N. Engl. J. Med. 348, 1625-1638 (2003).

7. Nakagawa, $\mathrm{H}$. et al. ER stress cooperates with hypernutrition to trigger TNFdependent spontaneous HCC development. Cancer Cell 26, 331-343 (2014).

8. Starley, B. Q., Calcagno, C. J. \& Harrison, S. A. Nonalcoholic fatty liver disease and hepatocellular carcinoma: a weighty connection. Hepatology 51, 1820-1832 (2010).

9. Calvisi, D. F. et al. Increased lipogenesis, induced by AKT-mTORC1-RPS6 signaling, promotes development of human hepatocellular carcinoma. Gastroenterology 140, 1071-1083 (2011).

10. Carroll, P. A. et al. Deregulated Myc requires MondoA/Mlx for metabolic reprogramming and tumorigenesis. Cancer Cell 27, 271-285 (2015).

11. Dang, C. V. Web of the extended Myc network captures metabolism for tumorigenesis. Cancer Cell 27, 160-162 (2015).

12. $\mathrm{Ma}$, C. et al. NAFLD causes selective CD4(+) T lymphocyte loss and promotes hepatocarcinogenesis. Nature 531, 253-257 (2016).

13. Li, J. et al. Lipid desaturation is a metabolic marker and therapeutic target of ovarian cancer stem cells. Cell Stem Cell 20, 303-314 e305 (2017).

14. Charni, M., Aloni-Grinstein, R., Molchadsky, A. \& Rotter, V. p53 on the crossroad between regeneration and cancer. Cell Death Differ. 24, 8-14 (2017)

15. Michalopoulos, G. K. \& DeFrances, M. C. Liver regeneration. Science 276, 60-66 (1997).

16. Dang, C. V. MYC on the path to cancer. Cell 149, 22-35 (2012).

17. Qu, A. et al. Role of Myc in hepatocellular proliferation and hepatocarcinogenesis. J. Hepatol. 60, 331-338 (2014).

18. Brodeur, G. M. Neuroblastoma: biological insights into a clinical enigma. Nat. Rev. Cancer 3, 203-216 (2003).

19. Qin, X. Y. et al. Transcriptome analysis uncovers a growth-promoting activity of orosomucoid-1 on hepatocytes. EBioMedicine 24, 257-266 (2017).

20. Qin, X. Y. et al. Prevention of hepatocellular carcinoma by targeting MYCNpositive liver cancer stem cells with acyclic retinoid. Proc. Natl Acad. Sci. USA 115, 4969-4974 (2018)

21. Muto, Y. et al. Prevention of second primary tumors by an acyclic retinoid, polyprenoic acid, in patients with hepatocellular carcinoma. Hepatoma Prevention Study Group. N. Engl. J. Med. 334, 1561-1567 (1996).

22. Qin, X. Y., Dohmae, N. \& Kojima, S. Reply to Yoshida: Liver cancer stem cells: Identification and lipid metabolic reprogramming. Proc. Natl Acad. Sci. USA 115, E6390-E6391 (2018).

23. Volmer, R., van der Ploeg, K. \& Ron, D. Membrane lipid saturation activates endoplasmic reticulum unfolded protein response transducers through their transmembrane domains. Proc. Natl Acad. Sci. USA 110, 4628-4633 (2013).

24. Hoadley, K. A. et al. Cell-of-origin patterns dominate the molecular classification of 10,000 tumors from 33 types of cancer. Cell 173, 291-304.e296 (2018).

25. Miyajima, A., Tanaka, M. \& Itoh, T. Stem/progenitor cells in liver development, homeostasis, regeneration, and reprogramming. Cell Stem Cell 14, 561-574 (2014).

26. Yamashita, T. et al. Discrete nature of EpCAM+ and CD90+ cancer stem cells in human hepatocellular carcinoma. Hepatology 57, 1484-1497 (2013).

27. Barretina, J. et al. The Cancer Cell Line Encyclopedia enables predictive modelling of anticancer drug sensitivity. Nature 483, 603-607 (2012).

28. Qin, X. Y. \& Kojima, S. Inhibition of stearoyl-CoA desaturase-1 activity suppressed SREBP signaling in colon cancer cells and their spheroid growth. Gastrointest. Disord. 1, 191-200 (2019).

29. Varga, J. \& Greten, F. R. Cell plasticity in epithelial homeostasis and tumorigenesis. Nat. Cell Biol. 19, 1133-1141 (2017).
30. Chen, B. P., Liang, G., Whelan, J. \& Hai, T. ATF3 and ATF3 delta Zip. Transcriptional repression versus activation by alternatively spliced isoforms. J. Biol. Chem. 269, 15819-15826 (1994).

31. Qin, X. Y. et al. Metabolome analyses uncovered a novel inhibitory effect of acyclic retinoid on aberrant lipogenesis in a mouse diethylnitrosamine-induced hepatic tumorigenesis model. Cancer Prev. Res. 9, 205-214 (2016).

32. Morrish, F. et al. Myc-dependent mitochondrial generation of acetyl-CoA contributes to fatty acid biosynthesis and histone acetylation during cell cycle Entry. J. Biol. Chem. 285, 36267-36274 (2010).

33. Park, E. J. et al. Dietary and genetic obesity promote liver inflammation and tumorigenesis by enhancing IL-6 and TNF expression. Cell 140, 197-208 (2010).

34. Niu, J. et al. Fatty acids and cancer-amplified ZDHHC19 promote STAT3 activation through S-palmitoylation. Nature 573, 139-143 (2019).

35. Yi, M. et al. Emerging role of lipid metabolism alterations in cancer stem cells. J. Exp. Clin. Cancer Res. 37, 118 (2018).

36. Lai, K. K. Y. et al. Stearoyl-CoA desaturase promotes liver fibrosis and tumor development in mice via a WNT positive-signaling loop by stabilization of low-density lipoprotein-receptor-related proteins 5 and 6. Gastroenterology 152, 1477-1491 (2017).

37. Pisanti, S., Picardi, P., D'Alessandro, A., Laezza, C. \& Bifulco, M. The endocannabinoid signaling system in cancer. Trends Pharmacol. Sci. 34, 273-282 (2013).

38. Guzman, M., Galve-Roperh, I. \& Sanchez, C. Ceramide: a new second messenger of cannabinoid action. Trends Pharmacol. Sci. 22, 19-22 (2001).

39. Mukhopadhyay, B. et al. Transcriptional regulation of cannabinoid receptor-1 expression in the liver by retinoic acid acting via retinoic acid receptorgamma. J. Biol. Chem. 285, 19002-19011 (2010).

40. Borradaile, N. M. et al. Disruption of endoplasmic reticulum structure and integrity in lipotoxic cell death. J. Lipid Res. 47, 2726-2737 (2006).

41. Ariyama, H., Kono, N., Matsuda, S., Inoue, T. \& Arai, H. Decrease in membrane phospholipid unsaturation induces unfolded protein response. J. Biol. Chem. 285, 22027-22035 (2010).

42. Liu, L. et al. Adaptive endoplasmic reticulum stress signalling via IRE1alphaXBP1 preserves self-renewal of haematopoietic and pre-leukaemic stem cells. Nat. Cell Biol. 21, 328-337 (2019).

43. Miharada, K., Sigurdsson, V. \& Karlsson, S. Dppa5 improves hematopoietic stem cell activity by reducing endoplasmic reticulum stress. Cell Rep. 7, 1381-1392 (2014).

44. Heijmans, J. et al. ER stress causes rapid loss of intestinal epithelial stemness through activation of the unfolded protein response. Cell Rep. 3, 1128-1139 (2013).

45. Wielenga, M. C. B. et al. ER-stress-induced differentiation sensitizes colon cancer stem cells to chemotherapy. Cell Rep. 13, 489-494 (2015).

46. Ma, M. K. F. et al. Stearoyl-CoA desaturase regulates sorafenib resistance via modulation of ER stress-induced differentiation. J. Hepatol. 67 979-990 (2017).

47. Dor, l., Namba, M. \& Sato, J. Establishment and some biological characteristics of human hepatoma cell lines. Gan 66, 385-392 (1975).

48. Fujise, $\mathrm{K}$. et al. Integration of hepatitis B virus DNA into cells of six established human hepatocellular carcinoma cell lines. Hepatogastroenterology $\mathbf{3 7}$ 457-460 (1990).

49. Cerami, E. et al. The cBio cancer genomics portal: an open platform for exploring multidimensional cancer genomics data. Cancer Discov. 2, 401-404 (2012).

50. Xia, J. G. \& Wishart, D. S. MetPA: a web-based metabolomics tool for pathway analysis and visualization. Bioinformatics 26, 2342-2344 (2010).

51. Gil, M., Samino, S., Barrilero, R. \& Correig, X. Lipid profiling using (1)H NMR spectroscopy. Methods Mol. Biol. 2037, 35-47 (2019). 